ISSN: 2637-773X

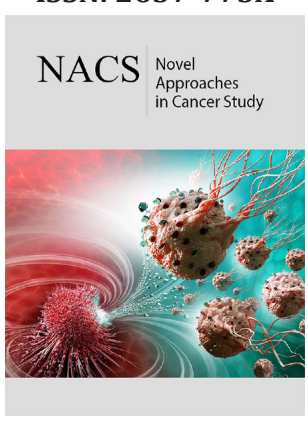

*Corresponding author: Kessel Boris, Division of General Surgery, Hashalom Street 2, Hadera, 38100, Israe

Submission: 诲June 24, 2021

Published: 酸July 07, 2021

Volume 6 - Issue 1

How to cite this article: Kessel Boris, Paran Maya, Shulman Katerina. Tumor-Agnostic Indications: Which Direction are We Going?. Nov Appro in Can Study. 6(1). NACS.000630. 2021. DOI: 10.31031/NACS.2021.06.000630

Copyright@ Kessel Boris, This article is distributed under the terms of the Creative Commons Attribution 4.0 International License, which permits unrestricted use and redistribution provided that the original author and source are credited.

\section{Tumor-Agnostic Indications: Which Direction are We Going?}

\author{
Kessel Boris ${ }^{1 *}$, Paran Maya ${ }^{1}$ and Shulman Katerina ${ }^{2}$ \\ ${ }^{1}$ Division of General Surgery, Israel \\ ${ }^{2}$ Carmel Medical Center, Israel
}

\section{Introduction}

The future belongs to technologies. It is certainly true in medicine, but particularly in clinical oncology. Immense improvements and advancements in many fields of medicine have already resulted in overall prolongation of life and improvement in its quality. However, there are still many aggressive, rapidly progressing malignant diseases that are associated with a short median survival time, despite all advances and development of high-resolution technologies, new drugs, and various treatment policies. In this work, we want to present, describe, and discuss the most promising, molecular advances in cancer research in our opinion that we believe would soon change the face of modern oncology.

All malignancies have historically been classified and treated based on tissue of origin, pathological features, and staging. First approved in 2017, a novel type of cancer therapy that is based on drugs or other substances designed to target cancer-specific genetic and molecular features, regardless of the cancer type or its anatomical location, is defined as tumor-agnostic therapy [1]. Biological markers - driven targeted therapies have transformed the horizons of the development of cancer therapeutics. Recent tissue-agnostic clinical trials represent a significant paradigm shift in the precision of cancer medicine. To date, despite multiple preclinical studies and gaining clinical experience, only a few biomarker-driven tissueagnostic indications received US Food and Drug Administration (FDA) approval. Primarily, this approach was used in immunotherapy and included Pembrolizumab (Keytruda) in microsatellite instability-high or mismatch repair deficient various solid tumors, such as colon, uterus, bladder, stomach, and other malignancies. The promising results in this unique population changed our reality. Currently, all metastatic colon cancer patients undergo routine microsatellite instability-high or mismatch repair screening and, when found positive, receive immunotherapy as a first-line treatment. Another agnostic indication is the use of Neurotrophic Receptor Tyrosine Kinase (NTRK) inhibitors in fusion-positive tumors. NTRK inhibitors are uses in a spectrum of different malignancies, including rare sarcomas, lung and thyroid cancers, and others. However, the reported incidence of positive NTRK patients is low, accounting for about $0-3 \%$ of all solid tumors [2]. Therefore, the screening for NTRK is currently indicated in patients who did not respond to existing lines of treatment.

Unfortunately, the overall prevalence of NTRK is low and thus the enrollment of eligible patients resulted in the recent lack of Phase III trials. The third novel tissue agnostic implementation is Tumor Mutational Burden (TMB). High TMB is a developing, independent biomarker for response and outcome prediction in immunotherapy in patients with various tumor types. High TMB is relatively frequently found in lung, urothelial, skin, and head \& 
neck tumors [3]. An additional significant element in the field of tumor-agnostic indications progress is drug approval. This process symbolizes another paradigm shift in drug development. Its progression is associated with several still controversial issues that should be considered, such as the amount of tissue needed for individual assays in different tumor types, additional clinical value, provided by such examinations, and the cost-benefit balance. The ethical question of biopsies performed for study purposes should also be addressed. Moreover, the clinical importance of the presence of a specific molecular marker is unequivocal. For example, lung tumors, associated with positive RET proto-oncogene fusion have a good probability of high response rates for specific biological drugs. On the other hand, the coexisting EGFR mutation may cause resistance to anti-EGFR treatment [4].

The two most common variations of tumor-agnostic therapy research are reflected in recent ongoing studies. An excellent example of one is a phase II study that evaluates the efficacy and safety of a biological drug (alectinib) in patients with solid locally advanced or metastatic tumors positive for Anaplastic Lymphoma Kinase (ALK). (https://clinicaltrials.gov/ct2/show/ NCT04644315). Patients with lung cancer and Cancer of Unknown Primary (CUP) are excluded. Another approach was presented the past year at The American Society of Clinical Oncology conference. The study includes patients with a variety of differentadvanced solid tumors, positive for multiple specific biomarkers, including Human Epidermal Growth Factor Receptor 2 (HER2), Phosphoinositide 3-Kinase (PI3K), Anaplastic Lymphoma Kinase (ALK), ProtoOncogene Tyrosine-Protein Kinase (ROS1), Protein Kinase B (AKT), Phosphatase and Tensin Homolog (PTEN), high tumor mutational burden (TMB), High Microsatellite Instability (MSI), and Deficient Mismatch Repair (dMMR). According to biomarkers presence, patients will be assigned to one of ten treatment arms to receive mono- or combination therapy with targeted agnostic treatments agents [5]. Results of these milestone studies will pave the way to a new era in clinical oncology.
Currently, molecular research of cancer biology is very complex and expensive, the clinical studies' design is complex, and the identification of resistance mechanisms is challenging. Moreover, the clinical significance of specific genome mutations remains unclear in the vast majority of cases. Each cancer cell includes an accumulation of multiple changes resulting from uncontrolled genomic material replication. It is still unknown whether a specific driver mutation that is found is responsible for the pathological pathways that allow modification of the existing therapeutic policies. However, the implementation of newest technologies such Next Generation Sequencing, identification of larger numbers of biological markers in addition to various studies, and the upcoming phase III clinical studies with enrollment of large numbers of patients in metastatic stage of disease make this research direction highly attractive and promising. Following the future expansion of tumor-agnostic indications will without a doubt, improve outcomes in oncologic patients.

\section{References}

1. Davis AA, McKee AE, Kibbe WA, Villaflor VM (2018) Complexity of delivering precision medicine: Opportunities and challenges. Am Soc Clin Oncol Educ Book 38: 998-1007.

2. Gatalica Z, Xiu J, Swensen J, Vranic S (2019) Molecular characterization of cancers with NTRK gene fusions. Mod Pathol 32(1): 147-153.

3. Samstein RM, Lee CH, Shoushtari AN, Hellmann MD, Shen R, et al. (2019) Tumor mutational load predicts survival after immunotherapy across multiple cancer types. Nat Genet 51(2): 202-206.

4. Bronte G, Ulivi P, Verlicchi A, Cravero P, Delmonte A, et al. (2019) Targeting RET-rearranged non-small-cell lung cancer: future prospects. Lung Cancer (Auckl) 10: 27-36.

5. Grothery A, Howland M, Hubbard L, Tania S, Anne M, et al. (2021) A study evaluating targeted therapies in participants who have advanced solid tumors with genomic alterations or protein expression patterns predictive of response (MyTACTIC). Journal of Clinical Oncology 39(15). 